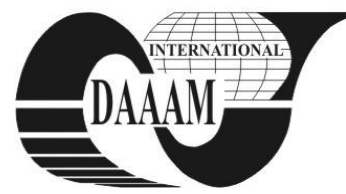

Annals of DAAAM for 2011 \& Proceedings of the 22nd International DAAAM Symposium, Volume 22, No. 1, ISSN 1726-9679 ISBN 978-3-901509-83-4, Editor B. Katalinic, Published by DAAAM International, Vienna, Austria, EU, 2011 Make Harmony between Technology and Nature, and Your Mind will Fly Free as a Bird Annals \& Proceedings of DAAAM International 2011

\title{
MUSIC LEARNING MANAGEMENT SYSTEM
}

\author{
SLOVAK, D[alibor] \& LATAL, P[etr]
}

\begin{abstract}
Music learning management system is being developed as a web-based support for teaching music theory. This part of the article is focused on basic description of the project. Music learning management system is there simply described with used technologies. In the next part of the article are approximated some CMS communications with publication and presentation. System is based on web technologies such as PHP, MYSQL and HTML with fully featured java-script jQuery API and WYSIWYG editor TinyMCE with useful plugins.

Key words: music LMS, CMS communications, VexFlow API cufon
\end{abstract}

\section{INTRODUCTION}

Music learning management system (in brief "MLMS") is being developed as solution for teaching music theory in modern way. MLMS has features such as front-end layout for teaching and backend system for administration. System is written with web based technologies such as PHP, HTML, MySQL and contains some plugins such as TinyMCE WYSIWYG editor used for the easy work with document writing. System has fully featured jQuery java-script library and special open-source API Vexflow which can display music staves.

This educational portal serves an environment for obtaining information in terms of fundamentals of music theory. The portal has the full-text search mechanism. Administrators can create any types of the tests that can be subsequently evaluated. The aim of this work was to create a Web interface that would allow students to register on the portal, classify students into the training courses, create components for tests managing and develop the simple interface for teaching with many features connected with the each course.

\section{CMS COMUNICATIONS}

CMS was developed to simplify the work as much as possible in creating of the new modules for communication between modules which have been secured against unauthorized access. Each page has been lightened by the administration of code that need not be loaded. The result is a system that communicates quickly and makes maximum use of optimized SQL queries.

Each module has its place on the ftp server in the /inc/ admin /. It is very important to have unique component containing a set of modifications (have to be named upravy.php) and a file containing error messages (have to be named hlasky.php). If you are missing these two files, you will be notified about this deficiency and asked to remedy it. It is good to have invented a meaningful file structure for adding, editing and general overview of the module.

Finally is necessary to have super admin account that allows authorized user to use the "Settings Module". This module is important for adding or editing the new modules into the system and has special forms for security features, classify features and graphical features.

\subsection{Language module}

The header file of language module is usually loaded once by system.php, which uses the php code filters for parsing country type and adds the value in the form of two lower case letter strings to the super global variable.(for example English has "en", Czech has "cs").

Then MySQL database queries the table "layout" and fetch data from the selected language to the website layout. If the language module cannot find a match with super global variable "lang", module will use the default language (in the case of teaching it is a Czech language). The database than fetches all data using the \$langcore["name_of_the_cell"] syntax and then simply display the value of the cell by the PHP function echo. Front-end web interface has simple switcher for language changing.

\subsection{Content creation}

Creating of the content in the MLMS system is very simple and efficient. This system in addition to their fundamental advantages such as creating and editing pages, deletion from the database, permitting or disabling content and other functions has also implemented multi-language support. In Figure 1 you can see the tree structure of the music portal website.

Layout of the website is designed for easy and quick user orientation in the content.

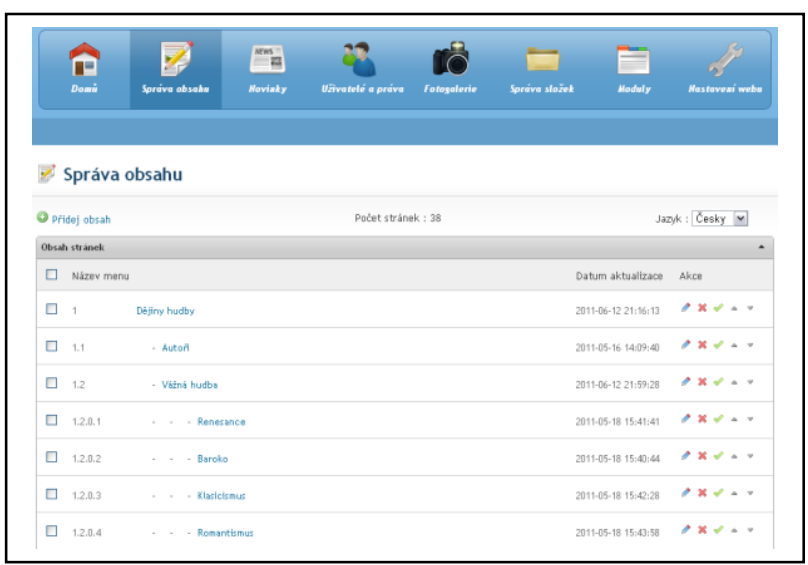

Fig. 1. Content management

The greatest benefits in terms of content creation, is a simple environment that avail functions that allows users to work in similar way as in MS Word. MLMS has an integrated WYSIWYG TinyMCE plugin with predefined style Office 2007 with many plugins, which facilitate the working. 


\subsection{Vexflow API}

VexFlow is the application programming interface for the music notation and can be used as a rendering engine for the various kinds of the online music learning tools, libraries, and applications. API is designed to run on HTML5 tag canvas with scalable vector graphics support.

It is important to note that the VexFlow is a low-level rendering API. Most applications wants to use something like VexTab which is a higher-level language for rendering guitar tablature and music notation.

VexFlow is written completely in JavaScript especially with jQuery API and using of HTML5 Canvas tag requires no external libraries or dependencies. For SVG support is necessary to include the Raphael JavaScript library to draw vector graphics on the MLMS site.

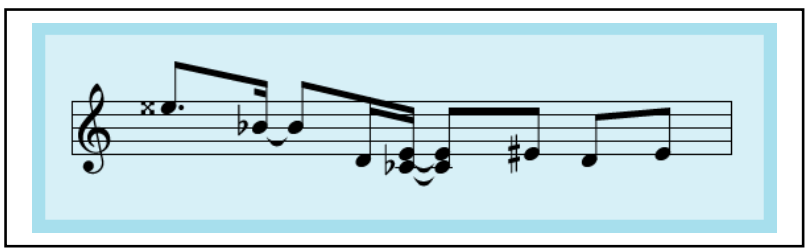

Fig. 2. Vexflow generated music stave

\subsection{Cufon}

Cufon JavaScript is a tool for replacing fonts on the web. Module uses two sets. One script is used for execution of Cufon API and one for replacement of fonts on Web. You can create almost any font in JavaScript and display them (The Cufon site http://cufon.shoqolate.com/generate/). Figure 3 shows example of cufon's generated font used on the MLSM site.

\section{HUDEBNI NAUKA}

Fig. 3. Cufon example h1 tag replacement

\section{PUBLICATION AND PRESENTATION}

The great advantage of the portal with the MLMS system is displaying the contents of pages with a uniform appearance. MLMS separates the system code from the text appearance and display it independently on the content. Therefore the authors do not care about the look but about the content.

An important factor is the portal site navigation (sitemap). This tool indexes all pages on the portal to the sitemap.xml file. Feature is ensured by an external XML Sitemap Generator (http://www.xml-sitemaps.com/). If that file exists, site is better indexed by Google robots and reason is that this site is better traced on searching sites.

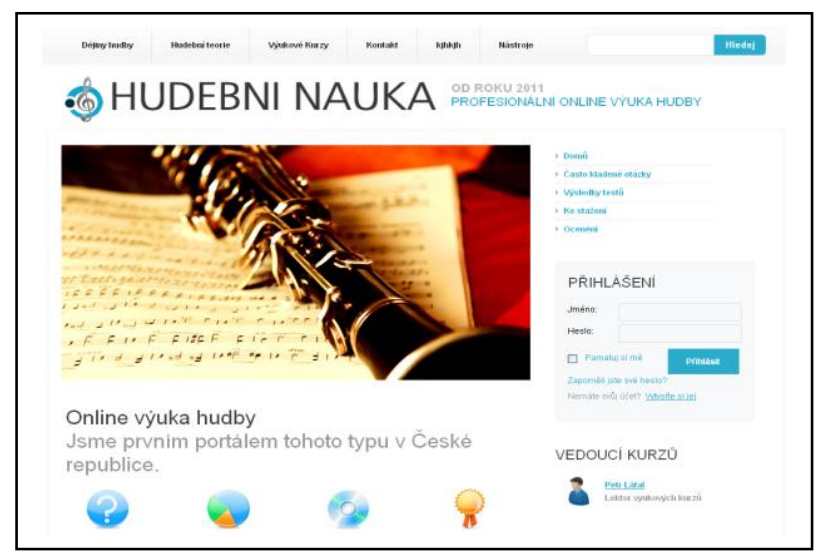

Fig. 4. Front-end web site presentation

\section{SYSTEM SECURITY}

Login to the administration is secured by the java-script md5 functions. This function encrypts the password to the md5 has form and then sent the submitted login form to the server. This way of submitting login forms ensures much greater system security since the data are transmitted in encrypted form and reduce the possibility of case of eavesdropping or deciphering of the seemingly simple form. This security does not work if you have java-script turned off. The system will alert you before log on and help you to setup java-script loading in browser. If the java-script will not to be set, you will be logged on, but not by the safe way.

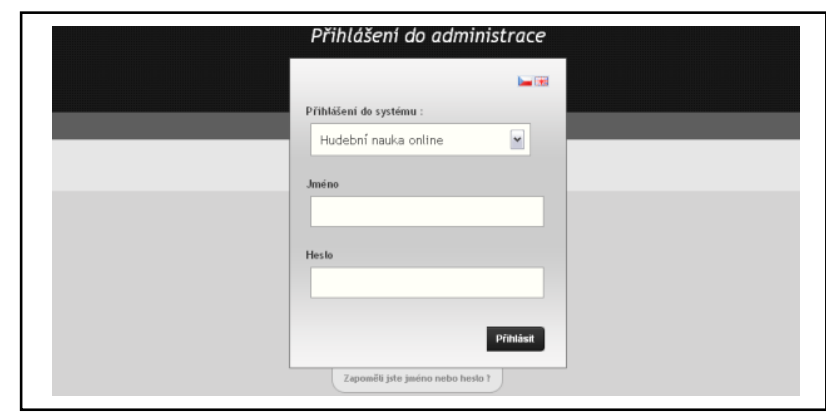

Fig. 5. Login to the administration

\section{CONCLUSION}

The aim of this article was to inform you about the unique learning portal of music theory. It was necessary to create a simple Multilanguage site for easy and quick navigation on the portal. It was necessary to develop the integral part of the system which was the MLMS administration system with the complex management teaching modules and the system for the generation of music staves.

We have described the basic information about portal and finally we would like to observe that the system is enhanced by the API jQuery that uses very effective asynchronous JavaScript with nice animations. We still work on the entire system and all links between the MLMS administration interface and the API VexFlow are still in progress. In the future, the system should be able to satisfy the ambitious teacher of music theory, including music MIDI player.

This was the first article from four which we want to describe. In the next article we would like to describe all modules connected with MLMS system and show their functions and in two other articles we should show the VexFlow core of the system with small tutorial how it works in real.

\section{REFERENCES}

Composite authors (2007), PHP 5, Computer Press, ISBN 978 80-251-1519-0, Brno

Castro, Elizabeth (2007). HTML : XHTML a CSS: web site creating. Computer Press, ISBN 978-80-251-1531-2, Brno.

Henk C.A : van TILBORG (2000). Fundamentals of cryptology, Kluwer Academic, ISBN 0-7923-8675-2, Norwell

Resig, John (2007). Javascript a AJAX : Modern programming of web applications, Computer Press, ISBN 978-80-2511824-5, Brno

Schlossnagle, Georgie (2004). PHP 5advanced programming, Computer Press, ISBN 80-86815-14-5, Brno.

Ullman, Larry (2004). HTML : PHP a MySQL, Computer Press, ISBN 80-251-0063-4, Brno

Zelinka, Ivan. (1999). Applied informatics, Editorial center, UTB, . ISBN 80-214-1423-5, Zlin 